\section{TRON BEAM TH SOLIDS}

non-equilibrium crystal growth phenomena using pulsed lasers, and (omputer modeling calculations of these rapid solidification effects were presented by P. Baeri (Catania, Italy). A. G. Cullis (RSRE, England) and J. Narayan (ORNL) presented results on phase transformations, defects and interfacial instability effects at these rapidly moving interfaces, and N. M. Johnson (Xerox) discussed electronic defects.

A joint session with the symposium on Metastable Materials Formation by Ion Implantation compared the effects of laser processing with those produced by ion bombardment. Significant insights into quenching aspects and metastable materials formation were provided in invited talks given by $\mathrm{S}$. Stritzker (KFA, Jülich, Germany), D. M. Follstaedt (Sandia), and J. E. E. Baglin (IBM).

\section{B. R. Appleton \\ Oak Ridge National Laboratory}

G. K. Celler

Bell Laboratories

Chairmen

\title{
MAGNETIC AND OPTICAL MATERIALS FOR INFORMATION STORAGE
}

Thirteen papers were presented at the Symposium on Magnetic and Optical Materials for Information Storage, covering new materials for recording heads, magnetic media, and optical disk data storage. Two new media for optical disks were detailed and critical analyses of existing materials were presented.

A necessary criterion for any optical recording medium is sensitivity sufficient to "write" at existing laser powers and wavelengths. But in some media, such as thin films of tellurium, high sensitivity brings a corresponding sacrifice of long term stability. This was clearly demonstrated in the report by $E$. LaBudde, which documented degradation in thin Te films under accelerated aging conditions. Several new media reported at the meeting do not suffer this corrosive degradation.

The earliest media recorded by ablation, a mechanism in which a pit is melted or vaporized by the laser spot. Ahn and coworkers from IBM described media based on amorphous films of noble metals and silicon. When heated by the laser "write" pulse, these sandwich films devitrify to form a crystalline silicide which differs in reflectivity from the unwritten background. Differences in reflectivity are sensed and read-out by a second optical beam.

Quite another approach to media was presented by R. E. Howard and H. G. Craighead of Bell Labs. They textured $\mathrm{Si}$ or $\mathrm{Ge}$ into submicron columnar structures by reactive ion etching. A write pulse melts the columns locally, creating a reflective spot. Read-out is based on the difference in reflectivity between the spot and the light-trapping columns.
Both the BTL and IBM media show promise of good archival stability in tests reported by their inventors.

But, as Alan Bell pointed out in his review, sensitivity and archivality are only two of the necessary features of an optical recording material. It must have low optical noise and a raw bit error rate of $<10^{-5}$ plus the feasibility of being fabricated at an acceptable cost.

The competition for optical disks is of course, magnetic media. In his keynote address, D. Speliotis clearly showed that magnetic recording has a long history of increasing bit densities and decreasing cost per bit. $\mathrm{He}$ emphasized that the implementation of vertical recording in which the easy axis of magnetization is perpendicular to the disk can give bit densities of $10^{4}$ per $\mathrm{cm}$. Still needed are improvements in materials beyond the present rate earthtransition metal magnetic alloys and thin film head materials.

A lively panel discussion at the close of the session brought forward many of the current issues facing this fast-emerging, materials-sensitive technology.

\section{Theodore Davidson}

Xerox Corporation

Chairman

On these pages appear brief reports by chairmen of some of the 1981 Annual Meeting symposia. Space does not permit all of the symposia highlights to appear in this issue of the Bulletin, and more reports will be published in the March-April issue. 